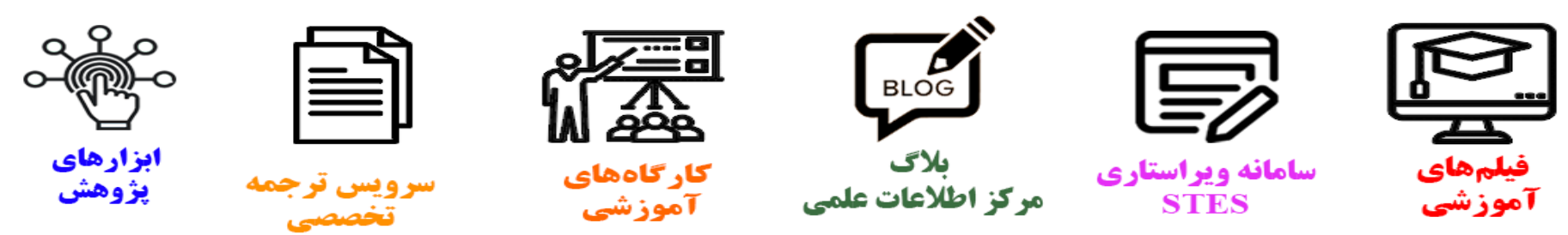

\title{
(c)
}

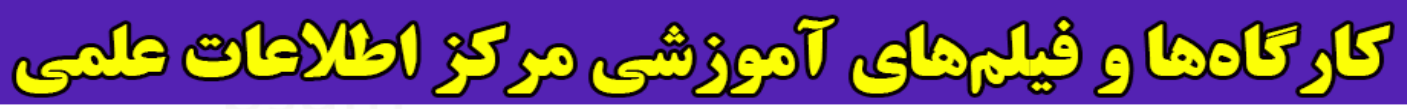
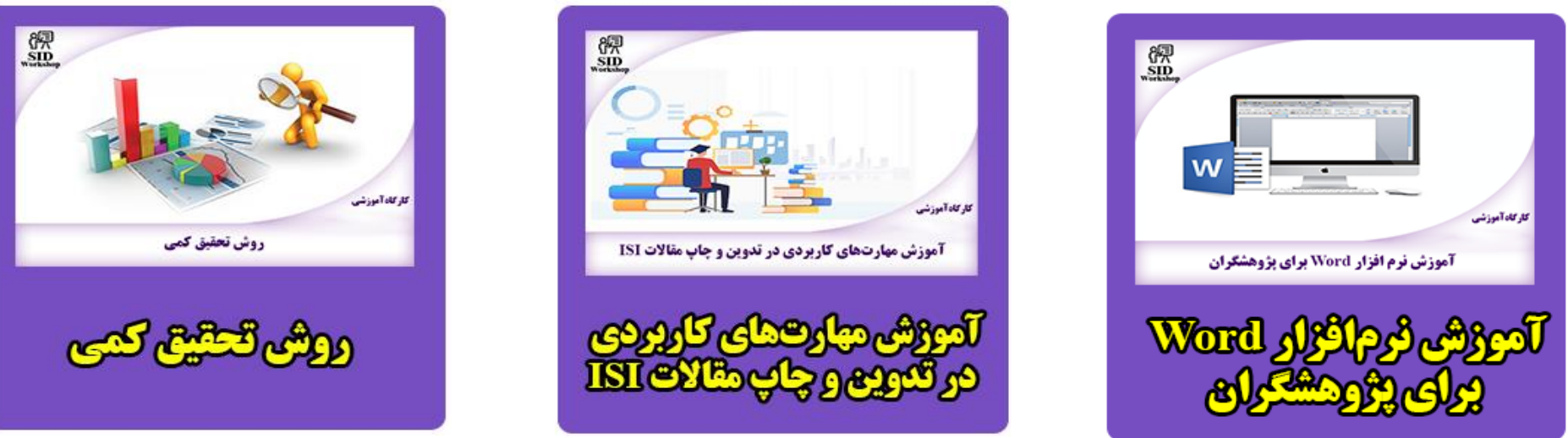


\title{
International Journal of Engineering
}

Journal Hom e page: ww w. ije.ir

\section{Suppression of Chaotic Behavior in Duffing-holmes System using Back-stepping Controller Optimized by Unified Particle Swarm Optimization Algorithm}

\author{
R. Gholipoura, A. Khosravi a, H. Mojallali*b \\ ${ }^{a}$ Faculty of Electrical and Computer Engineering, Babol University of Technology, Babol, Iran \\ ${ }^{b}$ Electrical Engineering Department, Faculty of Engineering, University of Guilan, Rasht, Iran
}

\section{PAPER INFO}

Paper history:

Received 07 October 2012

Received in revised form 23 January 2013

Accepted 28 February 2013

\section{Keywords:}

Duffing-holmes System

Control of Chaos

Back-stepping Controller

UPSO Algorithm

\section{$A B S T R A C T$}

The nonlinear behavior analysis and chaos control for Duffing-Holmes chaotic system is discussed in this paper. In order to suppress the irregular chaotic motion, an optimal back-stepping controller is designed. The back-stepping method consists of parameters with positive values. The improper selection of the parameters leads to inappropriate responses or even may lead to instability of the system. In this paper, the Unified particle swarm optimization (UPSO) algorithm is utilized to determine the convenient and optimal values of the parameters. The minimized objective function via UPSO algorithm is a weighted sum of the Integral of Time multiplied Absolute Error (ITAE) and squared control signal. Fast control of chaos in a very short time and having more limited control signal for this purpose, are the great advantages of the proposed controller. Numerical simulations show the high performance of this method for chaos elimination in Duffing-Holmes system.

\section{INTRODUCTION}

Chaos in control systems and controlling chaos in dynamical systems have both attracted much interest in recent years. A chaotic system has complex dynamical behaviors that possess some special features, such as excessive sensitivity to initial conditions, broad spectrums of Fourier transform, bounded and fractal properties of the motion in the phase space, and so on.

Chaotic phenomena can be found in many scientific and engineering fields such as biological systems, electronic circuits, power converters, chemical systems, and so on [1]. Chaotic systems have irregular, complex and unpredictable dynamic behavior. Since the pioneering work of Ott, et al. [2] proposed the well known OGY control method, the control of chaotic systems has been widely studied. Recently, quite a few techniques and approaches have been successfully applied to chaotic motion control under different conditions and requirements [3-5].

The back-stepping approach is one of the most popular nonlinear techniques of control design. It is

\footnotetext{
${ }^{*}$ Corresponding Author Email: mojallali@guilan.ac.ir (H.Mojallali)
}

capable of generating a globally asymptotically stabilizing control laws to suppress and synchronize chaotic system [6-14]. The idea of back-stepping design is to select recursively some appropriate functions of state variables as pseudo-control inputs for lower dimension subsystems of the overall system. Each backstepping stage results in a new pseudo-control design, expressed in terms of the pseudo-control designs from preceding design stages. When the procedure is terminated, a feedback design for the true control input results, which achieves the original design objective by virtue of a final Lyapunov function, which is formed by summing up the Lyapunov functions associated with each individual design stage.

Particle Swarm Optimization (PSO) is a swarm intelligence algorithm that is used mainly for numerical optimization tasks [15]. PSO gained increasing popularity in recent years due to its ability to solve efficiently and effectively a plethora of problems in science and engineering. Unified Particle Swarm Optimization (UPSO) was recently introduced as a modification of PSO that aggregates its local and global variant, combining their exploration and exploitation abilities without imposing additional requirements in 
terms of function evaluations. Convergence in probability was proved for a version of the new method and preliminary experimental results on both static and dynamic benchmark problems suggested that UPSO is superior to both the global and local variant of the standard PSO. In UPSO's main scheme, a new parameter, called the unification factor, was introduced in order to control the influence of the local and global PSO variants in the UPSO scheme [16]. UPSO Algorithm is used in this paper in order to determine the intelligent back-stepping controller parameters. In the proposed controller, the back-stepping method parameters are chosen such that the time response of system states converges to zero in a short time, i.e. the system chaos is controlled faster. Besides, more limited control signal is needed for stabilization of system states and chaos control.

The organization of this article is as follows. Section 2 describes the back-stepping method. PSO and UPSO are described in Section 3. The proposed optimal backstepping control design is given in Section 4. In Section 5 , simulation results are provided to validate the effectiveness of the proposed method. The conclusions of this paper are presented in Section 6.

\section{BACK-STEPPING METHOD}

Considering the following n-order system with strictfeedback form:

$$
\begin{aligned}
& \dot{x}_{i}=f_{i}\left(x_{1}, x_{2}, \ldots, x_{i}\right)+g_{i}\left(x_{1}, x_{2}, \ldots, x_{i}\right) x_{i+1}, 1 \leq \mathrm{i} \leq \mathrm{n}-1 \\
& \dot{x}_{n}=f_{n}\left(x_{1}, x_{2}, x_{3}, \ldots, x_{n}\right)+g_{n}\left(x_{1}, x_{2}, x_{3}, \ldots, x_{n}\right) u
\end{aligned}
$$

where $x \in R^{n}, u \in R$. With $f_{i}(0)=0$ and $g_{i}(0) \neq 0$ for $i=1, \ldots, n . f_{i}$ and $g_{i}$ are smooth functions, and are differentiable.

Step 1. Considering the first subsystem of (1), $x_{2}$ is taken as a virtual control input and choose:

$x_{2}=\frac{1}{g_{1}\left(x_{1}\right)}\left(u_{1}-f_{1}\left(x_{1}\right)\right)$

The first subsystem is changed to be $\dot{x}_{1}=u_{1}$. Choosing $u_{1}=-k_{1} x_{1}$ with $k_{1}>0$, the origin of the first subsystem $x_{1}=0$ is asymptotically stable, and the corresponding Lyapunov function is $V_{1}\left(x_{1}\right)=x_{1}^{2} / 2,(2)$ is changed to:

$x_{2}=\phi_{1}\left(x_{1}\right)=\frac{1}{g_{1}\left(x_{1}\right)}\left(-k_{1} x_{1}-f_{1}\left(x_{1}\right)\right)$

Step 2. Take $x_{3}$ as a virtual control input and the $\left(x_{1}, x_{2}\right)$ subsystem is changed to (5).

$x_{3}=\frac{1}{g_{2}\left(x_{1}, x_{2}\right)}\left(u_{2}-f_{2}\left(x_{1}, x_{2}\right)\right)$ $\dot{x}_{1}=f_{1}\left(x_{1}\right)+g_{1}\left(x_{1}\right) x_{2}$

$\dot{x}_{2}=u_{2}$

which is in the form of back-stepping method, so the control law $u_{2}$ is as follows:

$u_{2}=-\frac{\partial V_{1}}{\partial x_{1}} g_{1}\left(x_{1}\right)-k_{2}\left(x_{2}-\phi_{1}\left(x_{1}\right)\right)+\frac{\partial \phi_{1}}{\partial x_{1}}\left[f_{1}\left(x_{1}\right)+g_{1}\left(x_{1}\right) x_{2}\right]$

where $k_{2}>0$. This control law asymptotically stabilizes $\left(x_{1}, x_{2}\right)=(0,0)$ and Lyapunov function is as (7).

$V_{2}\left(x_{1}, x_{2}\right)=V_{1}\left(x_{1}\right)+\frac{1}{2}\left(x_{2}-\phi_{1}\left(x_{1}\right)\right)^{2}$

Substituting (6) into (4) gives:

$$
\begin{aligned}
& x_{3}=\phi_{2}\left(x_{1}, x_{2}\right)=\frac{1}{g_{2}\left(x_{1}, x_{2}\right)}\left[-\frac{\partial V_{1}}{\partial x_{1}} g_{1}\left(x_{1}\right)-k_{2}\left(x_{2}-\phi_{1}\left(x_{1}\right)\right)\right. \\
& \left.+\frac{\partial \phi_{1}}{\partial x_{1}}\left(f_{1}\left(x_{1}\right)+g_{1}\left(x_{1}\right) x_{2}\right)-f_{2}\left(x_{1}, x_{2}\right)\right]
\end{aligned}
$$

Step n: Actual control law $u$ where can asymptotically stabilize (1), is as follows:

$$
\begin{aligned}
& u=\frac{1}{g_{n}\left(x_{1}, \ldots, x_{n}\right)}\left[-\frac{\partial V_{n-1}}{\partial x_{n-1}} g_{n-1}\left(x_{1}, \ldots, x_{n-1}\right)-\right. \\
& k_{n}\left(x_{n}-\phi_{n-1}\left(x_{1}, \ldots, x_{n-1}\right)\right)+\frac{\partial \phi_{n-1}}{\partial x_{1}}\left(f_{1}\left(x_{1}\right)+\right. \\
& \left.g_{1}\left(x_{1}\right) x_{2}\right)+\ldots+\frac{\partial \phi_{n-1}}{\partial x_{n-1}}\left(f_{n-1}\left(x_{1}, \ldots, x_{n-1}\right)+\right. \\
& \left.\left.g_{n-1}\left(x_{1}, \ldots, x_{n-1}\right) x_{n}\right)-f_{n}\left(x_{1}, \ldots, x_{n}\right)\right]
\end{aligned}
$$

where $k_{n}>0$. This control law asymptotically stabilizes $\left(x_{1}, \ldots, x_{n}\right)=(0, \ldots, 0)$ and Lyapunov function is as $(10)$.

$V_{n}\left(x_{1}, \ldots, x_{n}\right)=V_{n-1}\left(x_{1}, \ldots, x_{n-1}\right)+\frac{1}{2}\left(x_{n}-\phi_{n-1}\left(x_{1}, \ldots, x_{n-1}\right)\right)^{2}$

\section{OPTIMIZATION METHOD}

3. 1. Particle Swarm Optimization Assume that each particle is considered in the $d$-dimensional space, $X_{i}(t)=\left(X_{i 1}(t), X_{i 2}(t), \ldots, X_{i D}(t)\right) \quad$ denotes the ith particle's position, $V_{i}(t)=\left(V_{i 1}(t), V_{i 2}(t), \ldots, V_{i D}(t)\right)$ denotes the ith particle's velocity. The best previous position of the ith particle $P_{\text {best }}$ is represented as $P_{i}(t)=\left(P_{i 1}(t), P_{i 2}(t), \ldots, P_{i D}(t)\right)$ and the best particle amongst all particles in the population $\mathrm{G}_{\text {best }}$ is represented as $P_{g}(t)=\left(P_{g 1}(t), P_{g 2}(t), \ldots, P_{g d}(t)\right)$. The 
velocity and position updating equations of the $\mathrm{PSO}$, for $\mathrm{d}=1,2, \ldots, \mathrm{D}$ are given below [17]:

$$
\begin{aligned}
& V_{i d}(t+1)=\chi *\left(V_{i d}(t)+c_{1} * r_{1} *\left(P_{i d}(t)-X_{i d}(t)\right)+\right. \\
& \left.c_{2} * r_{2} *\left(P_{g d}(t)-X_{i d}(t)\right)\right) \\
& X_{i d}(t+1)=X_{i d}(t)+V_{i d}(t+1)
\end{aligned}
$$

where $\chi$ is constriction factor, given by:

$$
\chi=\frac{2}{\left|2-\varphi+\sqrt{\varphi^{2}-4 \varphi}\right|}, \varphi=c_{1}+c_{2}, \varphi>4
$$

\subsection{Unified Particle Swarm Optimization} UPSO harnesses the two PSO variants (i.e. the local and global PSO variants) in a unified scheme that combines their exploration and exploitation capabilities. Let $X_{i}$ be the ith particle of the swarm, $g$ the index of the best particle in the whole swarm and $g_{i}$ the index of the best particle in the neighborhood of $X_{i}$. Also, let $\Psi_{i}(t+1)$ be the velocity update of $X_{i}$ for the global PSO variant, $L_{i}(t+1)$ the velocity update of $X_{i}$ for the local PSO variant, and the iteration counter. Then, from Equation (11), it holds that:

$$
\begin{aligned}
& \Psi_{i}(t+1)=\chi *\left(V_{i d}(t)+c_{1} * r_{1} *\left(P_{i d}(t)-X_{i d}(t)\right)+\right. \\
& \left.c_{2} * r_{2} *\left(P_{g d}(t)-X_{i d}(t)\right)\right) \\
& L_{i}(t+1)=\chi^{*}\left(V_{i d}(t)+c_{1} * r_{1}^{\prime} *\left(P_{i d}(t)-X_{i d}(t)\right)+\right. \\
& \left.c_{2} * r_{2}{ }^{\prime} *\left(P_{g_{i} d}(t)-X_{i d}(t)\right)\right)
\end{aligned}
$$

The aggregation of the search directions defined by Equations (14) and (15) results in the main UPSO scheme,

$$
\begin{aligned}
& U_{i}(t+1)=u * \Psi_{i}(t+1)+(1-u) * L_{i}(t+1), \mathrm{u} \in[0,1] \\
& X_{i d}(t+1)=X_{i d}(t)+U_{i d}(t+1)
\end{aligned}
$$

The parameter $\mathrm{u}$ is called the unification factor and it balances the influence of the global and local search directions. The standard global PSO variant is obtained by setting $\mathrm{u}=1$ in Equation (16), while $\mathrm{u}=0$ results in the standard local PSO variant. All intermediate values of $u \in(0,1)$ define composite UPSO variants that combine the exploration and exploitation properties of the global and local PSO variant. Indeed, the value $\mathrm{u}=0.5$ proved to be the best, with unification factors higher than 0.7 exhibiting poor performance [16]. The flowchart of the UPSO algorithm is given in Figure 1.

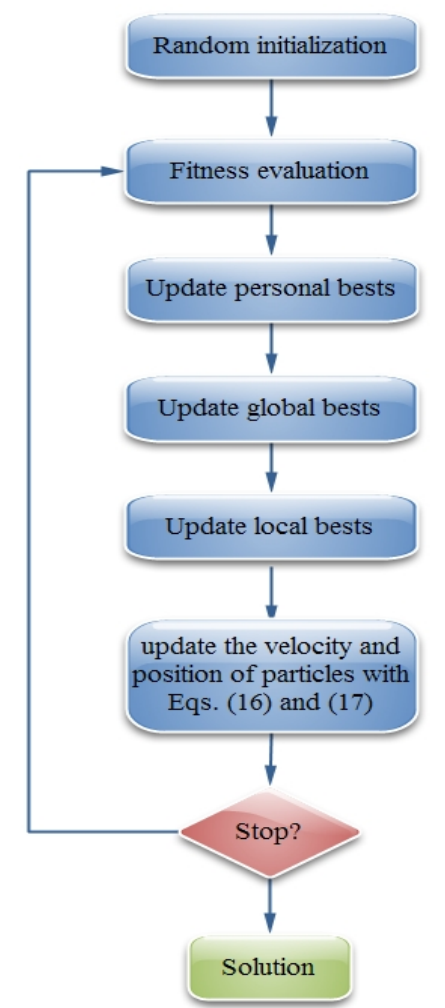

Figure 1. Flowchart of the UPSO algorithm

\section{PROPOSED OPTIMAL BACK-STEPPING CONTROLLER}

An optimal back-stepping controller is formed using UPSO Algorithm for the optimization of the backstepping controller key parameters. The proposed controller structure is shown in Figure 2. In this structure, $x_{i}(i=1,2, \ldots, n)$ are the state variables of the strict-feedback system, $\mathrm{k}_{\mathrm{i}}(\mathrm{i}=1,2, \ldots, \mathrm{n})$ the backstepping controller parameters and $\mathrm{u}$ is the control signal produced by the optimal back-stepping controller. In this structure, the state variables and the control signal are the inputs of the fitness function and establish the fitness function form. The output of the fitness function is the input of the UPSO. The UPSO Algorithm obtains the proper and optimal values of the parameters by minimizing the fitness function.

Here, the utilized objective function is similar to that of the reference [18]. The objective function is formulized as below:

$\left\{\begin{array}{l}J=\int_{0}^{t_{f}}\left[W_{1} t|E(t)|+W_{2} u^{2}(t)\right] d t \\ |E(t)|=\sum_{i=1}^{n}\left|\mathrm{e}_{i}(t)\right| \\ \mathrm{e}_{i}(t)=x_{i}(t)-x_{d i}\end{array}\right.$ 


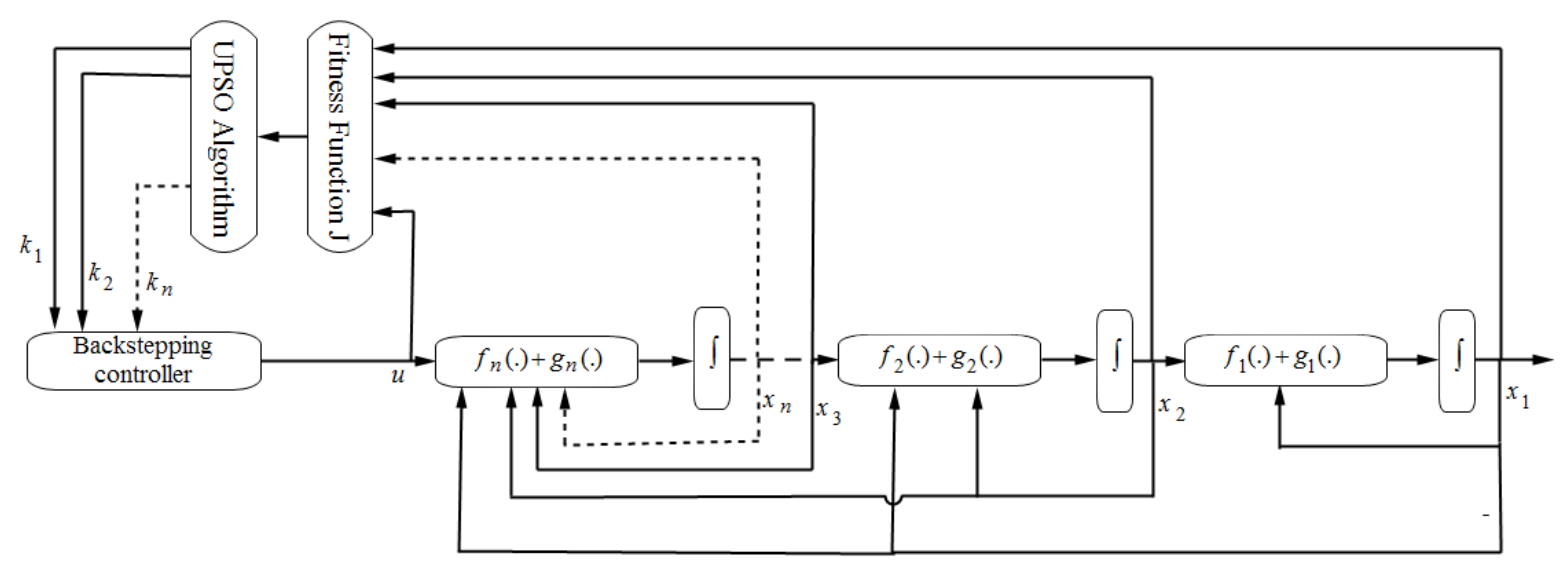

Figure 2. The structure of the proposed controller

where $t_{f}$ is the final time with respect to second, $u$ the control signal, $n$ the system degree, and $x_{i}(i=1,2, \ldots, n)$ are the system state variables, and $\mathrm{x}_{\mathrm{di}}(\mathrm{i}=1,2, \ldots, \mathrm{n})$ the desired trajectories of $x_{i}(i=1,2, \ldots, n)$. According to the goal of stabilizing and chaos control of the system, $x_{\mathrm{di}}$ $(\mathrm{i}=1,2, \ldots, \mathrm{n})$ is considered to be equal to zero. The fitness function form consists of two terms: The first term is the Integral of Time multiplied Absolute Error (ITAE) and the second term is the squared control signal. By minimizing the first term using UPSO Algorithm, the system states converge to zero in a short time, i.e. the system chaos is controlled in a short time. And by minimizing the second term, more limited control signal is required to control of chaos and to stabilize the system. The weights $\mathrm{w}_{1}$ and $\mathrm{w}_{2}$ are considered to be equal in this design procedure, in order to assume equal values for the two mentioned objectives, i.e. the minimization of the system error is as important as the limitation of the control effort. In fact, in the proposed controller, the back-stepping method parameters are chosen such that the time response of system states converges to zero in a short time, i.e. the system chaos is controlled faster. Besides, more limited control signal is needed for stabilization of system states and chaos control.

\section{AN ILLUSTRATED EXAMPLE}

5. 1. Duffing-holmes Chaotic System The point of interest considered in this study is a classical Duffing-Holmes chaotic system, and its nominal dynamic equations are described as [19]:

$\dot{x}_{1}(t)=x_{2}(t)$

$\dot{x}_{2}(t)=x_{1}(t)-0.25 x_{2}(t)-x_{1}^{3}(t)+0.3 \cos (t)$

where $x_{i}, i=1,2$ are the state variables, and $t$ is the time variable. For the initial condition $\left(x_{1}, x_{2}\right)=(0.2,0.2)$, the chaotic motion of the system is illustrated in Figures 3 and 4 .

\section{2. Controlling Duffing-holmes Chaotic System}

As shown in Figures 3 and 4, the system has a chaotic behavior, when no control signal is applied. In this section, the back-stepping method is utilized for the control of chaos of the Duffing-Holmes system. For this purpose, a control signal $u$ is added to Equation (19). The system (19) is rewritten, as following:

$$
\begin{aligned}
& \dot{x}_{1}(t)=x_{2}(t) \\
& \dot{x}_{2}(t)=x_{1}(t)-0.25 x_{2}(t)-x_{1}^{3}(t)+0.3 \cos (t)+u
\end{aligned}
$$

Back-stepping method is used to set states $x_{1}, x_{2}$ to the origin point $(0,0)$ via the control signal $u$ calculated with two steps. According to Section (1), the design procedure is as follows:

Step 1. $x_{2}$ is taken as (23) to construct the joint Lyapunov function (22) for (21).

$\dot{x}_{1}=x_{2}$

$V_{1}\left(x_{1}\right)=x_{1}^{2} / 2$

$x_{2}=\phi_{1}\left(x_{1}\right)=-k_{1} x_{1}$

Step 2. Final control input and Lyapunov function are given in (25) and (26) for (24).

$$
\begin{aligned}
& \dot{x}_{1}(t)=x_{2}(t) \\
& \dot{x}_{2}(t)=x_{1}(t)-0.25 x_{2}(t)-x_{1}^{3}(t)+0.3 \cos (t)+u \\
& u=-x_{1}(t)+0.25 x_{2}(t)+x_{1}^{3}(t)-0.3 \cos (t)- \\
& \left(1+k_{1} k_{2}\right) x_{1}(t)-\left(k_{1}+k_{2}\right) x_{2}(t) \\
& V_{2}\left(x_{1}, x_{2}\right)=\frac{1}{2} x_{1}^{2}+\frac{1}{2}\left(x_{2}+k_{1} x_{1}\right)^{2}
\end{aligned}
$$




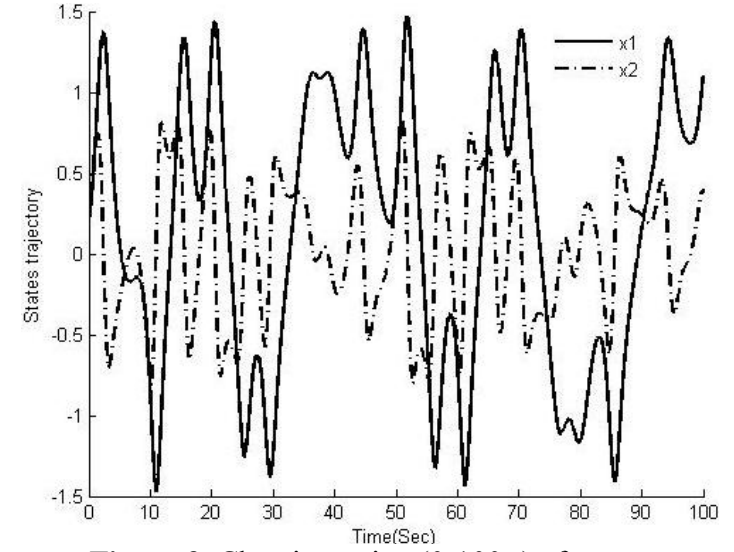

Figure 3. Chaotic motion (0-100 s) of states

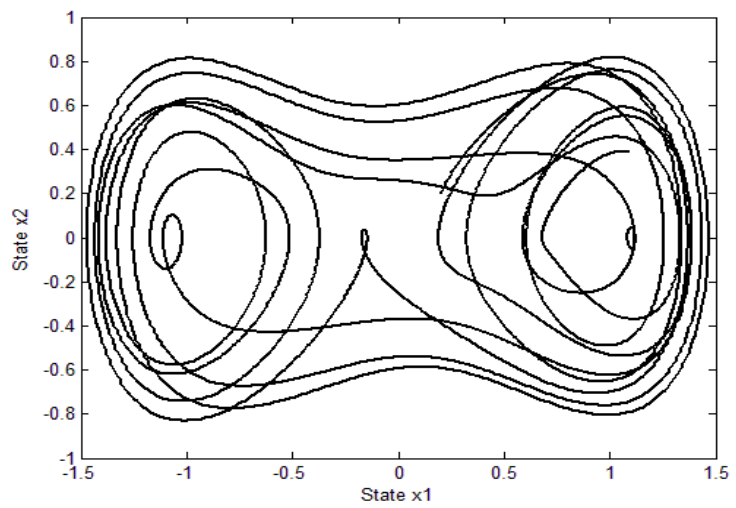

Figure 4. Chaotic attractor after $100 \mathrm{~s}$

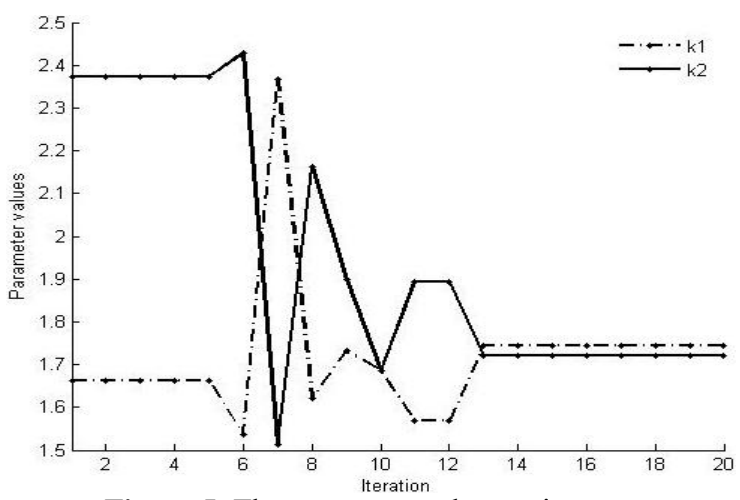

Figure 5. The parameter values trajectory

According to Equation (25), it is observed that the control signal consists of the parameters which are positive. The UPSO Algorithm obtains the proper values of the parameters via minimizing the fitness function. The parameters of the UPSO Algorithm are set to the following: $c_{1}=2.05, c_{2}=2.05, \chi=0.729, \mathrm{u}=0.5$ and population $\operatorname{size}=20$. For the computation of the local PSO components in UPSO, a neighborhood of radius 1 was used, in order to take full advantage of its exploration properties [16]. The sampling time in this simulation is 0.02 . In proposed controller, the searching ranges for the back-stepping parameters $k_{1}$ and $k_{2}$ are limited to $[0,10]$. The back-stepping parameters are obtained for 20 iterations. In this example $t_{f}$ is equal to 10 seconds. Besides, the weights $w_{1}$ and $w_{2}$ of fitness function are chosen as 0.5 . $n$ represents the system degree and is equal to 2 in this example. The parameters of back-stepping controller are obtained by using UPSO Algorithm, as follows: $k_{1}=1.7431, k_{2}=1.7194$. The search process of UPSO Algorithm for finding the parameters is shown in Figure 5. Besides, the fitness value obtained by the algorithm is 0.8435 . The trajectory of fitness variations with respect to algorithm iteration is shown in Figure 6. The time response of the states of Coullet system after applying the controller is shown in Figure 7. The controlled chaos of the system is demonstrated in Figure 8. Also, the control signal is illustrated in Figure 9. As shown in Figures 7 and 8, the UPSO Algorithm causes the time response of the states of the system converge to zero in a shorter time by minimizing the fitness function. In addition, according to Figure 9, it is observed that the proposed controller has created a limited control signal to chaos control of Coullet system.

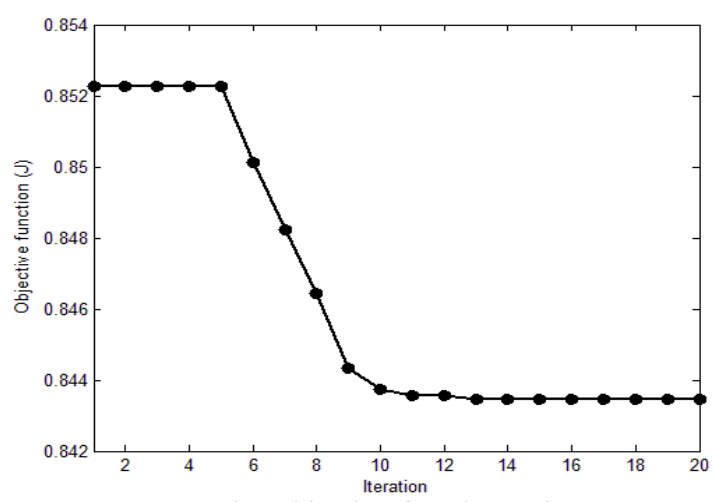

Figure 6. The Objective function trajectory

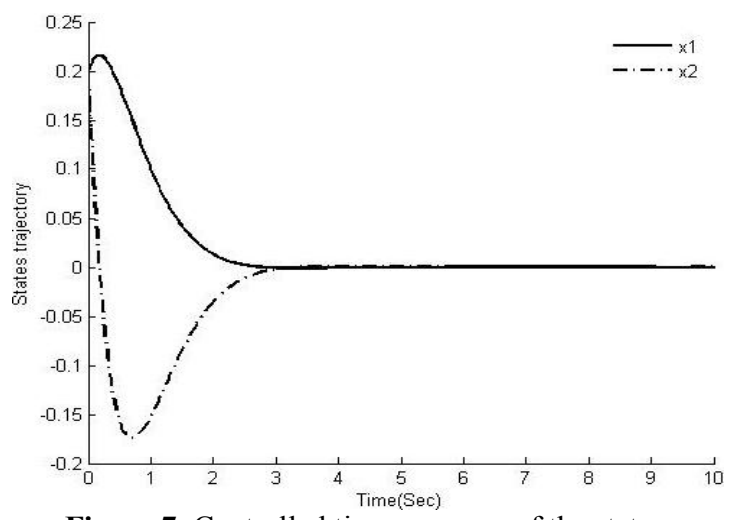

Figure 7. Controlled time response of the states 


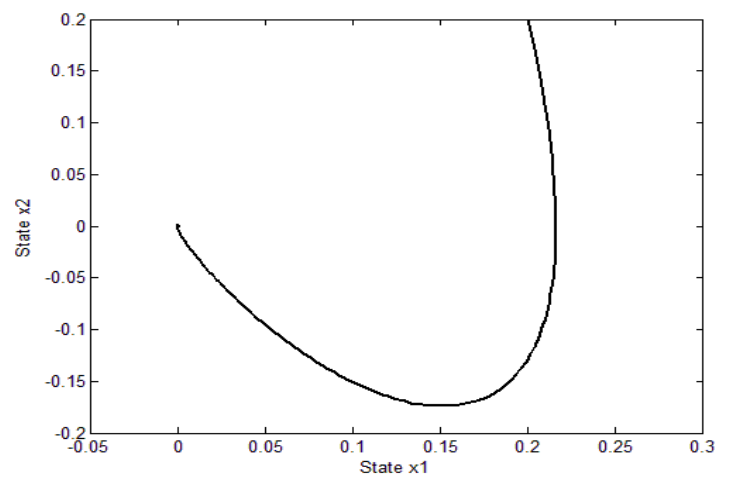

Figure 8. Controlled Chaotic attractor (0-100 s )

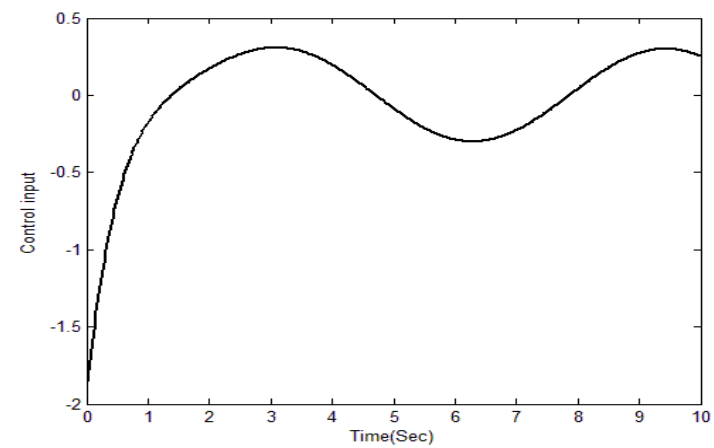

Figure 9. The control law u

5. 3. Discussion According to Figures 7 and 8 , it is observed that the UPSO Algorithm leads to fast time response of the system states by minimizing the fitness function. Whereas, if the values of the parameters were chosen by trial and error, the time response of the system states converged to zero in a much longer time. In fact, the intelligent back-stepping controller causes the system states to become stable in a shorter time and as a consequence, the system chaos is controlled in much shorter time. In addition, according to Figure 9, it is observed that the proposed controller produces a limited control signal for the chaos control of Duffing system. And it is because the control effort is applied in the proposed objective function. In fact, the UPSO Algorithm via the minimization of the objective function, causes the system states to become stable in a shorter time, i.e. the system chaos is controlled in a very short time and also, more limited control signal is needed to control chaos. Fast control of chaos in a very short time and having more limited control signal for this purpose, are the great advantages of the proposed controller.

\section{CONCLUDING REMARKS}

In this paper, an intelligent back-stepping controller has been proposed to control chaos in Duffing-Holmes system. In the proposed controller, the parameters of back-stepping method are determined automatically and intelligently by UPSO algorithm without trial and error. A weighted sum of the Integral of Time multiplied Absolute Error (ITAE) and squared control signal is the minimized fitness function via the UPSO algorithm. In the proposed controller, the back-stepping method parameters are chosen such that the time response of system states converges to zero faster, i.e. the system chaos is controlled in a short time. Besides, more limited control signal is needed for stabilization of system states and chaos control. The simulation results of Duffing Holmes chaotic system show the effectiveness of the proposed controller and the tuning process.

\section{REFERENCES}

1. Chen, G., "Controlling chaos and bifuractions in engineering systems", CRC press, (2000).

2. Ott, E., Grebogi, C. and Yorke, J. A., "Controlling chaos", Physical review letters, Vol. 64, No. 11, (1990), 1196.

3. Jiang, W., Guo-Dong, Q. and Bin, D., "Hळ variable universe adaptive fuzzy control for chaotic system", Chaos, Solitons \& Fractals, Vol. 24, No. 4, (2005), 1075-1086.

4. Hua, C., Guan, X. and Shi, P., "Adaptive feedback control for a class of chaotic systems", Chaos, Solitons \& Fractals, Vol. 23, No. 3, (2005), 757-765.

5. Guo, H., Lin, S. and Liu, J., "A radial basis function sliding mode controller for chaotic lorenz system", Physics Letters A, Vol. 351, No. 4, (2006), 257-261.

6. Lu, J. and Zhang, S., "Controlling chen's chaotic attractor using back-stepping design based on parameters identification", Physics Letters A, Vol. 286, No. 2, (2001), 148-152.

7. Park, J. H., "Synchronization of genesio chaotic system via back-stepping approach", Chaos, Solitons \& Fractals, Vol. 27, No. 5, (2006), 1369-1375.

8. Bowong, S. and Moukam Kakmen, F., "Chaos control of uncertain chaotic systems via back-stepping approach", Journal of Vibration and Acoustics, Vol. 128, No. 1, (2006), 21-27.

9. Yassen, M., "Chaos control of chaotic dynamical systems using back-stepping design", Chaos, Solitons \& Fractals, Vol. 27, No. 2, (2006), 537-548.

10. Chen, F., Chen, L. and Zhang, W., "Stabilization of parameters perturbation chaotic system via adaptive back-stepping technique", Applied Mathematics and Computation, Vol. 200, No. 1, (2008), 101-109.

11. Wu, Z.-J., Xie, X.-J., Shi, P. and Xia, Y.-q., "Back-stepping controller design for a class of stochastic nonlinear systems with markovian switching", Automatica, Vol. 45, No. 4, (2009), 9971004.

12. Ma, R. and Zhao, J., "Back-stepping design for global stabilization of switched nonlinear systems in lower triangular form under arbitrary switchings", Automatica, Vol. 46, No. 11, (2010), 1819-1823.

13. Shi, H., "A novel scheme for the design of back-stepping control for a class of nonlinear systems", Applied Mathematical Modelling, Vol. 35, No. 4, (2011), 1893-1903.

14. $\mathrm{Hu}, \mathrm{Q}$, $\mathrm{Xu}, \mathrm{L}$. and Zhang, A., "Adaptive back-stepping trajectory tracking control of robot manipulator", Journal of the Franklin Institute, Vol. 349, No. 3, (2012), 1087-1105. 
15. Eberhart, R. and Kennedy, J., "A new optimizer using particle swarm theory", in Micro Machine and Human Science, MHS'95., Proceedings of the Sixth International Symposium on, IEEE, (1995), 39-43.

16. Parsopoulos, K. E. and Vrahatis, M. N., "Parameter selection and adaptation in unified particle swarm optimization", Mathematical and Computer Modelling, Vol. 46, No. 1, (2007), 198-213.

17. Clerc, M. and Kennedy, J., "The particle swarm-explosion, stability, and convergence in a multidimensional complex space", Evolutionary Computation, IEEE Transactions on, Vol. 6, No. 1, (2002), 58-73.

18. Pan, I., Das, S. and Gupta, A., "Tuning of an optimal fuzzy pid controller with stochastic algorithms for networked control systems with random time delay", ISA Transactions, Vol. 50, No. 1, (2011), 28-36.

19. Yan, J.-J., "Design of robust controllers for uncertain chaotic systems with nonlinear inputs", Chaos, Solitons \& Fractals, Vol. 19, No. 3, (2004), 541-547. 


\title{
Suppression of Chaotic Behavior in Duffing-holmes System using Back-stepping Controller Optimized by Unified Particle Swarm Optimization Algorithm
}

\author{
R. Gholipour a A. Khosravi a, H. Mojallalib
}

a Faculty of Electrical and Computer Engineering, Babol University of Technology, Babol, Iran

${ }^{b}$ Electrical Engineering Department, Faculty of Engineering, University of Guilan, Rasht, Iran

\section{PAPER INFO}

Paper history:

Received 07 October 2012

Recivede in revised form 23 January 2013

Accepted 28 February 2013

\section{Keywords:}

Duffing-holmes System

Control of Chaos

Back-stepping Controller

UPSO Algorithm

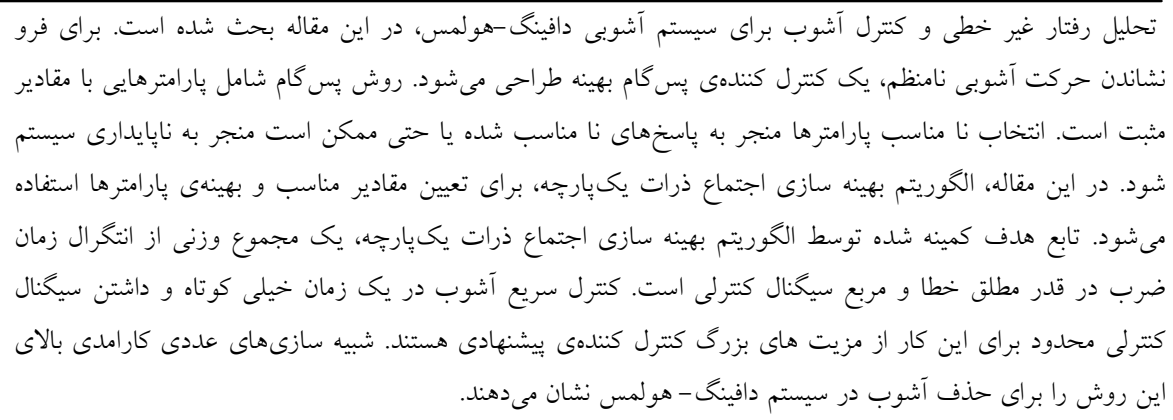




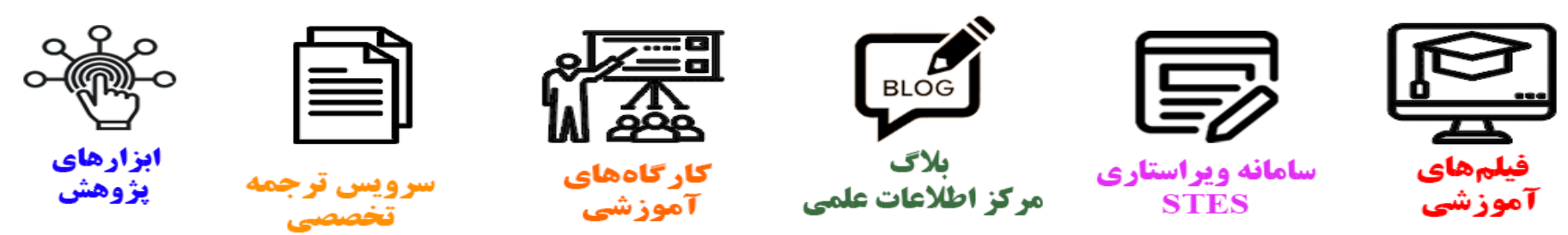

\section{(c)}

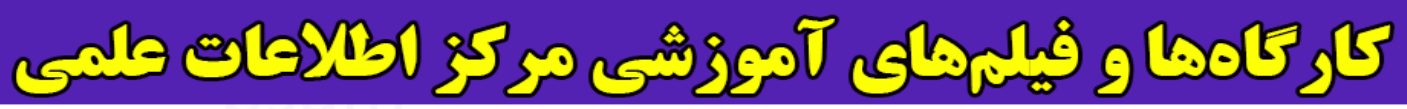
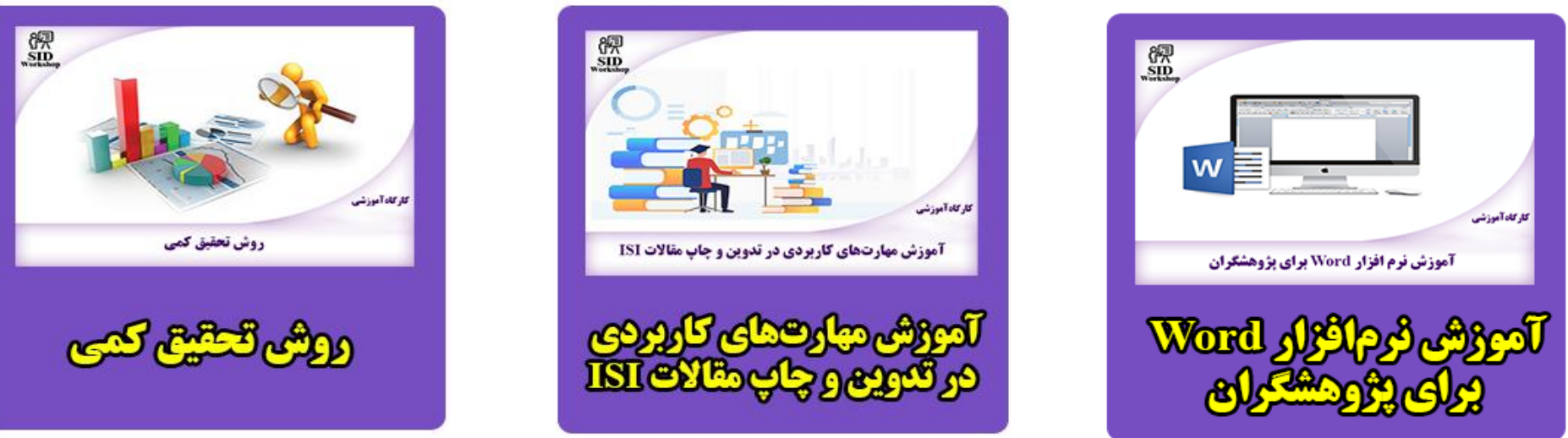